\title{
Insidious Phrenic Nerve Involvement in Postpolio Syndrome
}

\author{
Tomihiro Imai and Hiroyuki Matsumoto
}

\begin{abstract}
A 49-year-old woman with amyotrophic sequelae of poliomyelitis experienced progressive left upper limb weakness and breathing discomfort while walking that had developed over one year prior to presentation. She had flaccid quadriplegia which was more marked in the left upper limb, with the C4- and C5-innervated muscles being most severely affected. Chest radiographs double exposed at maximal inspiration and expiration revealed poor respiratory movements in the left hemidiaphragm. Phrenic nerve conduction study demonstrated conspicuous diaphragmatic paralysis on the left side. The electrophysiological study supports radiological findings and provides useful evidence for insidious phrenic nerve involvement in postpolio syndrome.
\end{abstract}

Key words: postpolio syndrome, phrenic nerve palsy, respiratory failure, phrenic nerve conduction, diaphragmatic action potential

(DOI: 10.2169/internalmedicine.45.1657)

\section{Introduction}

We describe a patient with postpolio syndrome (PPS), in whom unilateral phrenic nerve involvement was finally diagnosed by phrenic nerve conduction study. Respiratory insufficiency, which is common in patients with PPS (1), may be associated with sleep apnea syndrome. Although respiratory complications may be expected to correlate with kyphoscoliosis and diaphragmatic paralysis demonstrated by radiology, these symptoms often have an insidious onset and usually develop without particular signs on clinical and radiological examinations (1).

\section{Case Report}

A 49-year-old woman with limb amyotrophy attributed to polio sequelae presented with progressive left upper limb weakness and breathing discomfort while walking. The symptoms had developed approximately 1 year prior to presentation, 43 years after the initial polio. In a routine follow-up examination for polio sequelae conducted at the age of 46, laboratory tests including phrenic nerve conduction showed no respiratory abnormalities (Fig. 1). A second assessment of respiratory function was conducted at our institution to investigate the presenting symptoms.
At the age of 5, the patient had paralytic anterior poliomyelitis with quadriplegia, which was more marked in the left upper limb. She recovered progressively, with sequelae including moderate paresis and amyotrophy of proximal muscles of the left upper limb, but no conspicuous weakness of respiratory muscles.

New onset of muscle weakness in the left upper limb was diagnosed as PPS after electrophysiological and neuroradiological studies ruled out neurological conditions of motor neuron disease, cervical radiculomyelopathy and peripheral neuropathy. Electromyographical study revealed a poor interference pattern in the atrophic muscle of the left upper limb. No spontaneous activity was recorded from the neurogenic atrophic muscles at rest. Laboratory data did not indicate other underlying medical disorders such as diabetes, collagen disease, other major viral illnesses, and exposure to toxic agents.

Pulmonary function tests detected no restrictive abnormality (total lung capacity was 2.95 liter, $117 \%$ of predicted). Forced expiratory maneuver was completed in less than one second. The $\mathrm{PCO}_{2}$ and $\mathrm{O}_{2}$ were $37.2 \mathrm{mmHg}$ and 94.5 $\mathrm{mmHg}$, respectively, in room air.

Although a routine posteroanterior radiograph of the thorax showed normal findings, chest radiographs exposed at maximal inspiration and expiration revealed poor movements in the left hemidiaphragm. We performed a phrenic 

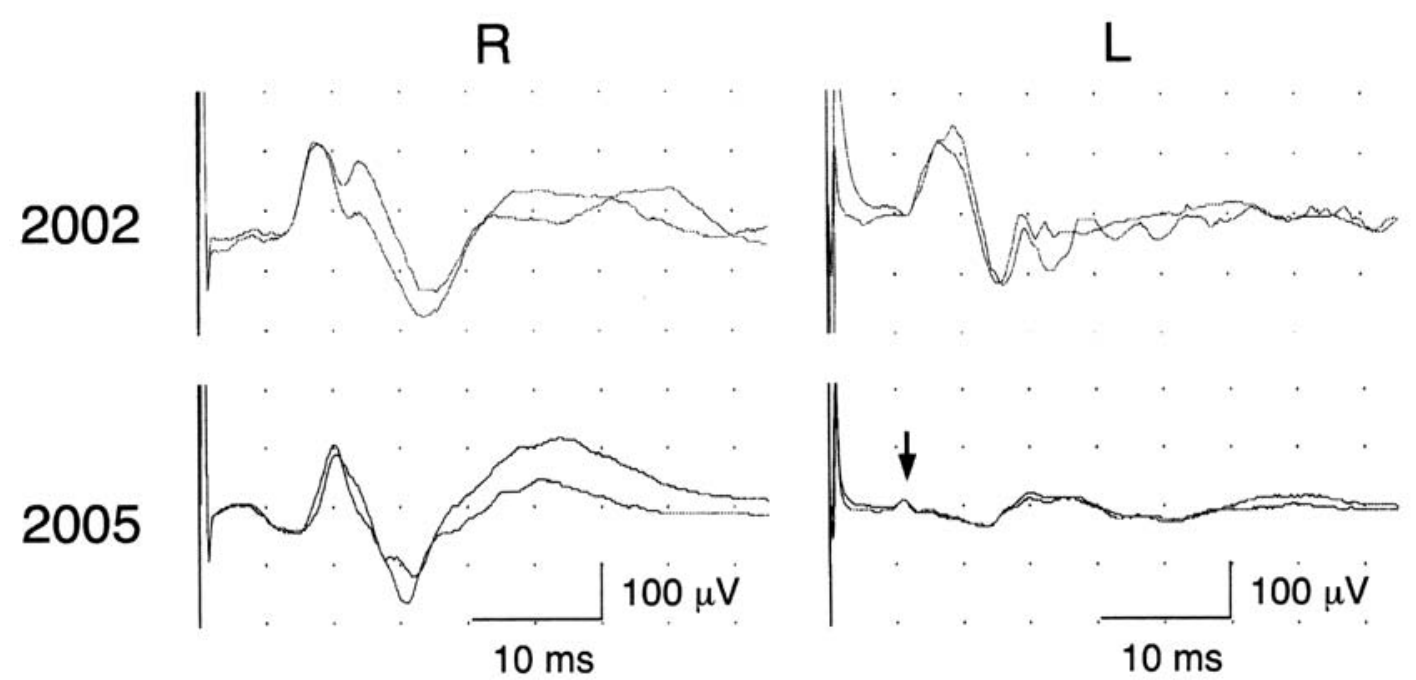

Figure 1. Diaphragmatic action potentials (DAPs) generated by phrenic nerve stimulation at the supraclavicular fossa. Note marked depression of left DAPs compared to the data obtained 3 years earlier, in contrast to unchanged DAPs on the right side. The arrow indicates the onset of left DAP.

nerve conduction study and compared with the results of the previous study done at the age of 46 . The diaphragmatic action potentials (DAPs) were recorded using a Viking IV electromyograph (Nicolet Biomedical, Madison, WI, USA). The recording method and normal data have been described in our previous study (2).

The right DAPs showed normal biphasic waves with a latency of $7.3 \mathrm{~ms}$ and amplitude of $150 \mu \mathrm{V}$, which were essentially unchanged compared with the results obtained three years ago. On the other hand, the left DAPs were significantly attenuated $(<50 \mu \mathrm{V})$ with no latency shifts, which had developed over the previous three years (Fig. 1). It was difficult to determine the onset of the left DAPs in the second examination because of unduly low amplitudes. We judged that a very small negative deflection at $6 \mathrm{~ms}$ (arrow in Fig. 1) was the onset of DAP, after reconfirming reproducibility of the results. These temporal changes in DAPs demonstrated late progressive involvement of phrenic motor neurons in PPS.

\section{Discussion}

New onset of weakness, sometimes accompanied by muscle atrophy, is the most significant neurological problem af- ter polio, and has been referred to as PPS. The weakness is usually asymmetric and can be proximal, distal or patchy. Autopsy studies have demonstrated inflammation in the spinal cord of subjects with PPS (3). However, the inflammatory process is not fully elucidated even though some mechanisms such as persistent poliovirus infection, immunemediated syndrome, and response to degenerating neurons have been proposed (3). Another hypothesis is that an excessive metabolic stress on the remaining motor neurons over many years eventually results in the dropout of the new nerve terminals and eventually the motor neurons themselves (4). The latter hypothesis is consistent with statistical data indicating that weakness is more likely to develop later in previously affected muscles than in clinically unaffected muscles, as seen in the present case. Neuronal loss may be localized mainly in the left cervical cord, especially C3-5 that provides the phrenic nuclei (5) and motor neurons innervating the proximal muscles of the upper limb.

It remains unclear whether the incomplete phrenic nerve palsy and breathing discomfort while walking are independent or associated in this case. Follow-up study is warranted to elucidate the temporal relationship between respiratory insufficiency and the course of phrenic nerve palsy which may remain unilateral or become bilateral in the future.

\section{References}

1. Lane DJ, Hazleman B, Nichols PJR. Late onset respiratory failure in patients with previous poliomyelitis. Q J Med 43: 551-568, 1974.

2. Imai T, Yuasa H, Kato Y, Matsumoto H. Aging of phrenic nerve conduction in the elderly. Clin Neurophysiol 116: 2560-2564, 2005.

3. Jubelt B, Drucker J. Poliomyelitis and the post-polio syndrome. In: Motor Disorders, Younger DS, Eds. Lippincott Williams \&
Wilkins, Philadelphia, 1999: 381-395.

4. Grimby G, Stalberg E, Sandberg A, Sunnerhagen KS. An 8-year longitudinal study of muscle strength, muscle fiber size, and dynamic electromyogram in individuals with late polio. Muscle Nerve 21: 1428-1437, 1998.

5. Rouval RV, Pal GP. Location of the phrenic nucleus in the human spinal cord. J Anat 195: 617-621, 1999.

(C) 2006 The Japanese Society of Internal Medicine http://www.naika.or.jp/imindex.html 